\title{
Fuzzy Neuro Approach for Active Queue Mangament
}

\author{
Vishal Chandra \\ Al, SGVU \\ Jaipur,Rajasthan,India
}

\author{
Savita shiwani, Ph.D \\ IT, SGVU \\ Jaipur,Rajasthan,India
}

\begin{abstract}
Out internet is growing dressily day and night. One of the major problem in internet is to reduce the loss of packet during transmission. There are various nodes or devices so called router, switch, hub, and repeater are there between sender and receiver. Since the responsibility of network layer to transfer the packet to source to destination. Router is a network layer device, which route the data packets. Router give the path to the packets so that they reach the destination with minimum time and minimum loss. Each and every router has a buffer space in which packets wait for processing. This is known as queue of packets. These are also known as active queue, because they are alive they are waiting for processing they wait due to router processor speed, incoming packet rate, outgoing packet rates also due to difference of band width between incoming and outgoing packets. There are various active queue management mechanism or algorithm were developed such as RED, BLUE, SFB, CHOKE, PURPLE etc. but have their pros and cons. In this paper active queue management is done by using fuzzy logic. Basically fuzzy logic is used for decision making. Same here which packet have to drop or which not will be decided by fuzzy logic and some parameters which are used in this paper. There will be some membership graphs and rules and Mamdani mechanism use to get appropriate answer. Also a dataset is used to validate the theory.
\end{abstract}

\section{Keywords}

AQM, Active Queue Management, Fuzzy logic, fuzzy theory, membership graph, congestion control

\section{INTRODUCTION}

In last few decades, the number of computer user, internet user and network traffic have grown explosively amount. The Transmission Control Protocol (TCP) congestion control theory was introduced by Van Jacobson in the Internet in the years of 1980's. The present Internet service only provides the best effort service within various circumstances. In the current best effort services of internet, provides processing of traffic as quickly as possible, but there is no guarantee of timeline and actual delivery of packet from senders to receivers. Quality of service is one of the most important factor for developing new internet services. There are two most important models has proposed by Internet Engineering Task Force (IETF) for providing quality of service in the Internet are:

\section{Integrated Services (Intserv) and}

2. Differentiated Services (Diffserv)

In the Integrated Services (Intserv) model, network resources are reserved for each individual flow of packet. This model uses the Resource Reservation Protocol (RSVP)) as the signaling protocol as TCP (Transmission Control Protocol. The concept of the Differentiated Services (Diffserv) for traffic is into two different classes and give them differentiated mechanism. Each and every router in the Internet have two classes of algorithms for congestion control:

\section{Queue management and}

\section{Scheduling.}

The tasks of queue management algorithms is to manage the length of packets queues by dropping them whenever it is necessary or appropriate, on the other hand the scheduling algorithms focused on the packets which will be sent in sequel and they used primarily allocation of bandwidth among packet flows. The First-In First-Out (FIFO) is one of the most common packet scheduling algorithm. Dropping of packets is considered as the notification of implicit network congestion. Routers which are used in internet, queues mechanism to incoming packet rates. When the rate of incoming packets is higher than that of the router's outgoing packet rate, then the queue size will abruptly increase; the amount of increase in incoming packets will ultimately exceed an available buffer space or size. When the buffer space is full, rest of packets will be dropped. In the Tail-Drop or Drop Tail (DT) policy, which is the one of the most worldwide dropping policy, in this policy when packet arrives and feel the queue size is full, then packets will be dropped. Researches says that the DT mechanism one of the worse TCP's mechanism for congestion control, results to poor a performance network.

Congestion control concern about controlling traffic entry into a communication network and outgoing to the network. Congestion control concepts is techniques and mechanisms that can prevent congestion or avoid congestion that can prevent congestion or remove congestion when it happen. Conventional congestion control scheme are divided into following two broad categories:

\section{1) Open-Loop Congestion Control}

This helps in preventing congestion before it happens. Congestion can be prevented at either the source or destination by using Retransmissions Policy, Acknowledgment Policy and Discard Policy.

\section{2) Closed-Loop Congestion Control}

This helps to remove congestion after happening of congestion in network. It uses following techniques to remove congestion like Choke Point, Back Pressure, Explicit Signaling ,Implicit Signaling and to remove congestion.

There are two kinds of congestion control:

a) Router based (which uses AQM) and

b) End host based ( which uses TCP)

The main and most important target of congestion control is to gain efficiency, minimum queue size, means maximum utilization, and minimum packet drops while providing fair access to all the sources and destinations. There are usually two approaches for congestion control:

1) Network-centric congestion control and

2) End system congestion control 
First approach is end system congestion control, in this mechanism senders can detect the congestion and react to it according to the congestion control algorithms and congestion control mechanisms. A simple example of this approach TCP, whenever a packet is dropped, and the sender assumes that congestion may occurred in anywhere in the network and it reduces the sending rate of packets. Whenever a packet is successfully delivered, sender increase their rate of sending packets. The second approach is network-centric based congestion control mechanism. The main idea behind this mechanism is that routers have more or almost all information about the state, topology of the network, routers can be useful in detecting congestion and can take decision of congestion control. Routers can measure the congestion level, to do this it compare input traffic to capacity and by estimating at the queue size; thus, the algorithm sends feedback as soon as it noticed that the queue length is growing exponentially. Therefore, queue length shouldn't be as large as in the previous approach. One of the simplest practical example of network-centric congestion control is Active Queue Management.

\section{ACTIVE QUEUE MANAGEMENT}

AQM whose full name is Active Queue Management represent the class of algorithms that are designed to provide an improved queuing mechanisms for routers. These mechanisms are called active because routers dynamically send congestion signal to sources even before the queues overflow; in both type either explicitly, means by marking packets (e.g. Explicit Notification of Congestion) or implicitly, by dropping packets using algorithm. There are mainly two designs considered in any Active Queue Management (AQM)

1. How to measure the congestion in the network and

2. How that measured congestion is used to calculate the packet drop probability.

Queue based AQMs are coupled with congestion notification rate of the queue size. The Active Queue Management Algorithms (AQMs) currently employed over the Internet, such as Drop tail and RED becomes under this category. Another example of a queue based Active Queue Management Algorithm (AQM) is BLUE algorithm.

The Active Queue Management (AQM) mechanism approach is used for informing the sender for the anticipation of congestion before the happening of congestion in routers' buffer overflow. By using Active Queue Management (AQM) approaches, the senders are warned in advance about congestion status so that they react accordingly. The RED algorithm mechanism approach is randomization of packets to overcome not only full queue problems but also the lockout problem effectively without making any changes at the senders end. The task of RED algorithm is to avoid drop-tail and queue length at each router. In RED algorithm packets are dropped randomly from which are come from various TCP networks at different times. Thus the TCP mechanism for flow control for these TCP connection will reduce their sending rate at different time. Active Queue Management algorithm used not only queuing delay but also load on node to determine packet drop probability to examine and then the delay load/delay Controller (LDC) which can estimate accurately load/delay and also anticipate the congestion. The other mechanism for controlling congestion is a Closed-Loop Congestion Control (CLCC) mechanism scheme is on top of an existing Active Queue Management (AQM) mechanism to attain accurate, fair, precise bandwidth distribution among concurrent non-responsive and responsive traffic, has been proposed. The new mechanism which his active queue management scheme has the advantages such that it does not require to estimate the level of traffic responsiveness. An $\mathrm{AQM}$ is the evaluation framework schemes has been proposed. The averaging queuing effect of on REDs gives ability to avoid a bias against busty traffic, to reduce queue size.

The Control messages from the routers' are preferred to pass to the during queue congestion time for these reason which are as follows:

1. Network topology is update by control message by router. Therefore, they prevent data packet to be transmitted through broken paths.

2. There are two things in network connection oriented and connection less Data packets are fully "connection oriented", they guaranteed that to delivery to their destinations by TCP protocol. In opposite to, control messages they are "connectionless"; means, if they dropped message, will not be retransmitted again.

3. The size of control message is very small compared to data packet. Inany ad-hoc based routing protocol, the size of control message is 64 bytes and size of data packet is 512 bytes, therefore the control message takes small space than data packet in the routers' queue and processing will be fast in the node or router.

\subsection{Random Early Drop (RED)}

Random Early Detection was designed for achieving four objectives they are as follows

1. Minimizing packets loss and queuing delay

2. To Avoid global synchronization for sources

3. Gaining high utilization links, and

4. Remove biases against busty sources.

The main idea behind Random Early Drop (RED) mechanism of active queue management is to detect emergence of congestion as early as possible and to send notification of congestion to the sender and receiver, allowing them to throttle down their transmission rates before router's queues in the network overflow and packets will be dropped.

\subsection{Flow Random Early Drop (FRED)}

FRED (Flow Random Early Drop) is a modifiedalgorithm of RED, which uses active-per-flows accounting to make different dropping decisions from RED for connections of with different bandwidth usages by the senders and receivers. The task of FRED algorithm is only to keeps track the of flows that have packets in the router' buffer queue, this supports that the cost of FRED algorithm is directly proportional to the buffer size inside routers' and FRED algorithm is independent of the total number of flow.

\section{BLUE}

BLUE an advanced type of active queue management algorithm that manage congestion control by link utilization and packet loss utilization history instead of capacity of queue. BLUE usages a single probability, $P_{m}$, parameter to mark (or drop) packets drop probability. When the routers' queue is continually dropping packets due to routers' buffer overflow, BLUE increases $P_{m}$, probability his factor or probability mark thus increasing the rate at which it sends 
back to the congestion notification or allow dropping packets. Conversely, if the queue becomes empty or if the link is idle, BLUE decreases Pm marking probability or probability mark. This effectively allows BLUE to "learn" the correct rate it needs to send back congestion notification or dropping packets. It also works as flow control algorithm because it sends congestion notification and packet dropping information to senders or sending nodes.

\section{CHOKe}

As in any active queue management algorithm, CHOKe algorithm or technique is differentially, it penalizes the nonresponsive and unfriendly flows using routers' queue buffer availability information of each flow through network. CHOKe estimate the average availability of routers' buffer queue with the help of the FIRST IN FIRST OUT (FIFO) buffer using an exponential algorithm which moving average, just as RED algorithm does. CHOKe also use to marks two thresholds on the buffer,

1. A maximum threshold max $_{t h .}$ and

2. A minimum threshold $\min _{t h}$

a). Basic $\mathrm{CHOKe}(\mathrm{CHOKe})$ : It works exactly as described CHOKe algorithm for active queue management in the above, but it, choose only single packet at a single time to compare with the arriving packet.

b). Adaptive $\mathrm{CHOKe}$ (A-CHOKe): A most sophisticated way to implement $\mathrm{M}-\mathrm{CHOKe}$ is to let algorithm automatically choose the appropriate number of packets chosen from routers' buffer. In A-CHOKe or adaptive $\mathrm{CHOKe}$, it is to partition the interval between minimum threshold minth and the maximum threshold maxth

b). Multi-drop CHOKe (M-CHOKe): In M-CHOKe or multidrop-CHOKe algorithm ,in this algorithm $\mathrm{m}$ packets are chosen from the routers' buffer to compare with the arriving packet, and drop those packets that must have the same flow ID as the arriving packet. It is important to notice that choosing more than one candidate packet improves CHOKe's performance

\section{PROPOSED MODEL}

In this proposed model first fuzzy system decide which packet to stay, wait or drop at the same time fuzzy system train the neural network to act in future when it get data similar type of data it get in future about 10000000 data are used to train neural network.

Even trained system fails then again fuzzy system train it to get best result.

The proposed model is combination of two technologies they are as follows:

$$
\begin{aligned}
& \text { 1. Fuzzy logic } \\
& \text { 2. Neural network }
\end{aligned}
$$

For solving problem of handling active queue at router end.

Fuzzy logic is used for decision making and neural network used for training to the system, both neural network and fuzzy logic have various variants but in this paper only simple approach is used to solve router's problem which packet should drop or which to stay or which to processed.

In this proposed model there are two systems fuzzy system and neural network system.

1. First this paper shows how fuzzy logic work

2. Second neural network work

Fuzzy logic - it is multivalued logic, fuzzy means vague, imprecise, cannot be determine by using crisp set or logic. Suppose we want to rank 10 beautiful girls how we measure their beurty.in this situation fuzzy comes.

It has membership function and inference rules and inference engines like Mamdani Sugeno. Since this paper deals with the approach of neural network and fuzzy logic that's why whole description is not necessary.

Neural network- it is basically used for training a system by some certain algorithm or weight and bias.

In this paper there are five linguistic variables were taken
1. Size of incoming packet
2. Incoming bandwidth
3. Outgoing bandwidth
4. Available queue size
5. Delay at router

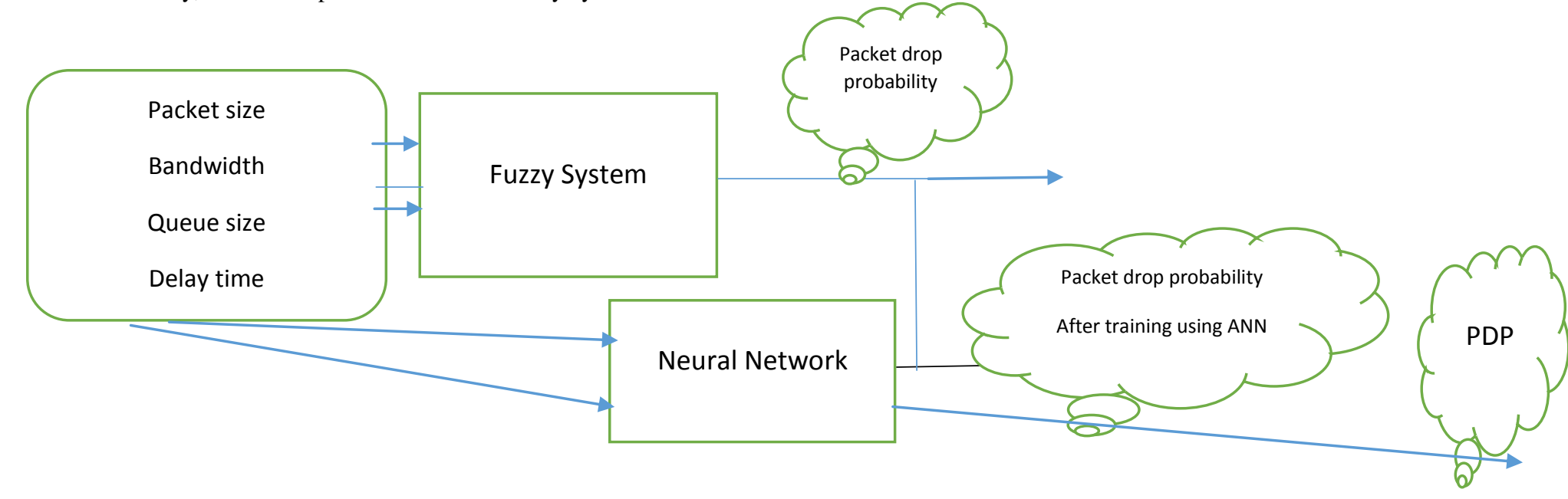


1. Size of incoming packets

Size of incoming packets: in sending and receiving of data is important because it will occupy more space than other also it is not good for low bandwidth system. If size of incoming packet is small the chance to congestion is low because processing time will required low but when big size data is transmitted then processing them very tough

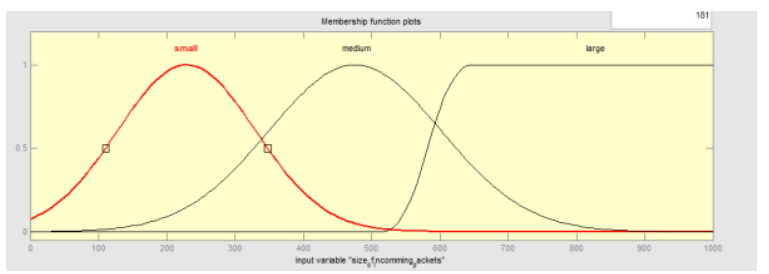

2. Incoming bandwidth

It is the capacity of incoming network that how much packet can travel from this media. If incoming rate than outgoing rate then bottleneck problem will occur so incoming bandwidth is also the part of solution of active queue management

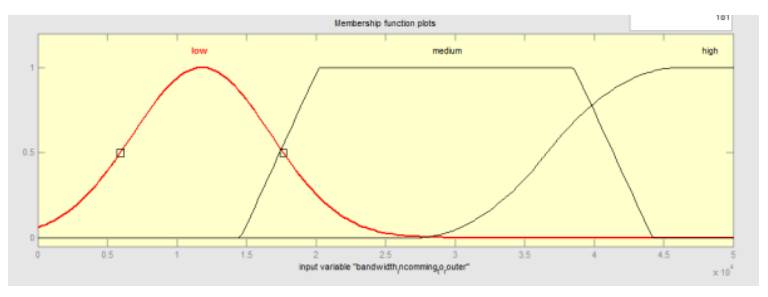

3. Outgoing bandwidth

It is the capacity of outgoing network that how much packet can travel from this media. If incoming rate is low than outgoing rate high then bottleneck problem will occur so outgoing bandwidth is also the part of solution of active queue management

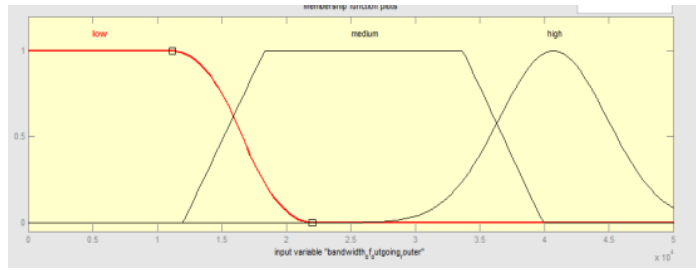

4. Available queue size

The routers' buffer size is limited when packets are in routers' buffer then it is known as active queues, because they are ready to go they may not be drop.

$\mathrm{Bu}$ in active queue management the active queue may also drop to prevent congestion. This paper helps to determine which packet should wait, drop, go.

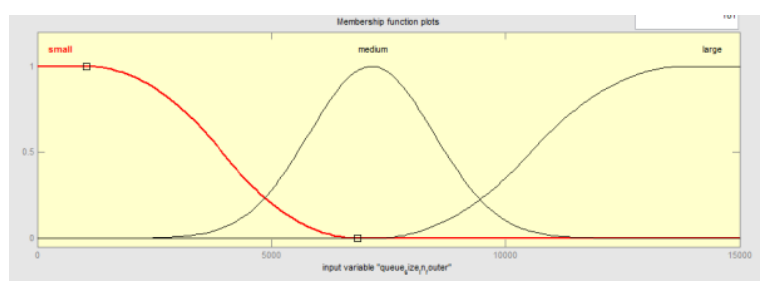

5. Delay at router

There are various reason for delay at router end, this may be due to processor speed packet size bandwidth

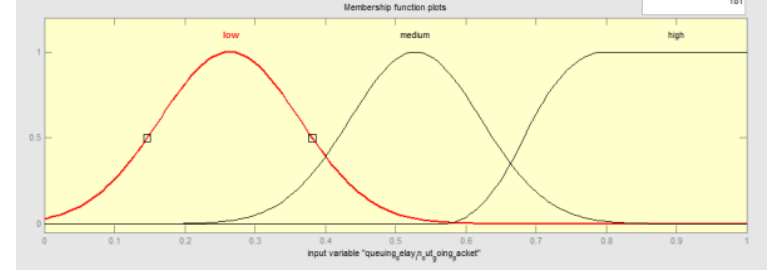

6. Surface view

This surface curve show the dependencies of two over on variable like bandwidth, packet size, delay

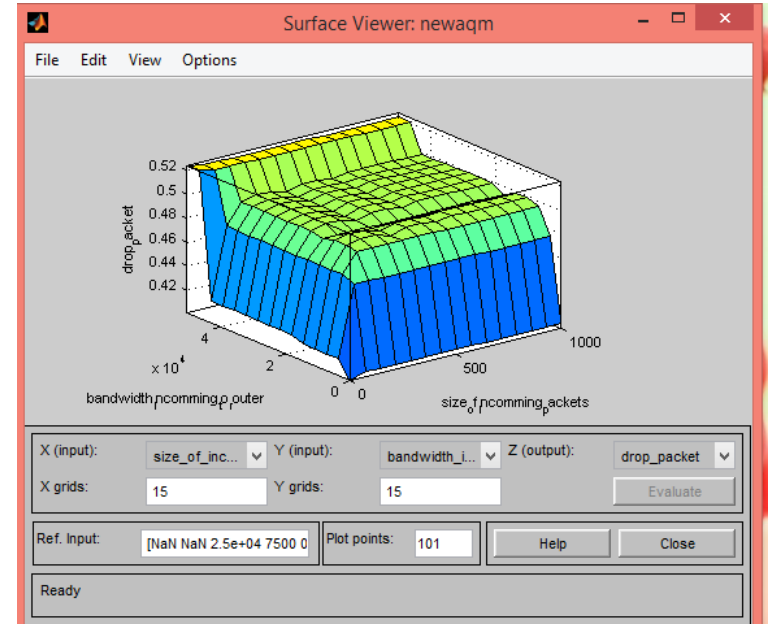

7. There are 243 rules are written to show the accuracy of the model.

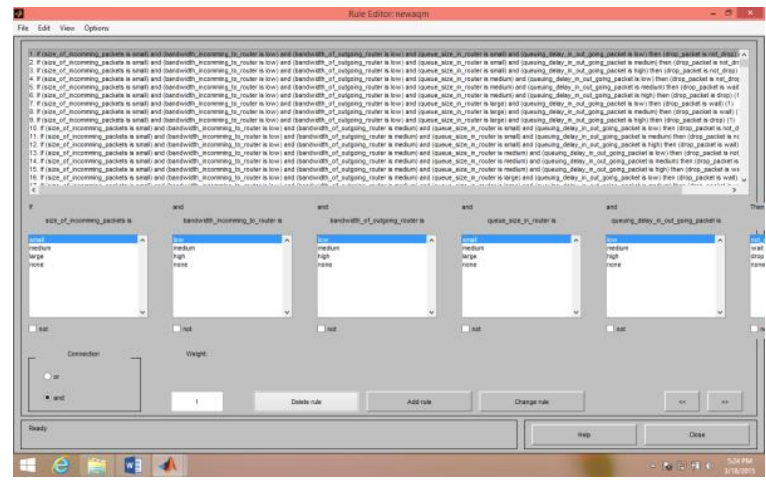

Performance

Regression
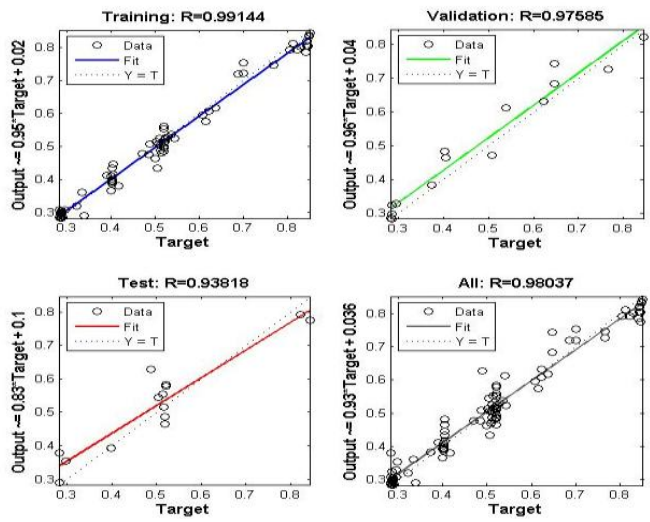
States
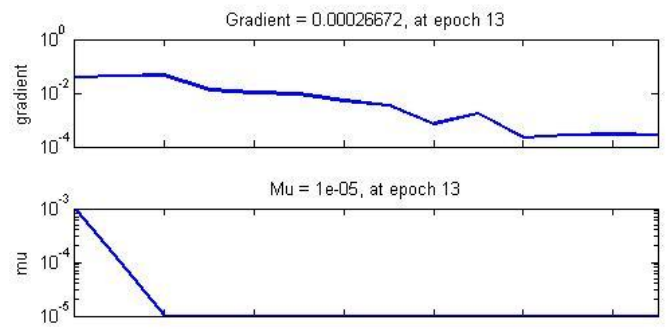

Validation Checks $=6$, at epoch 13

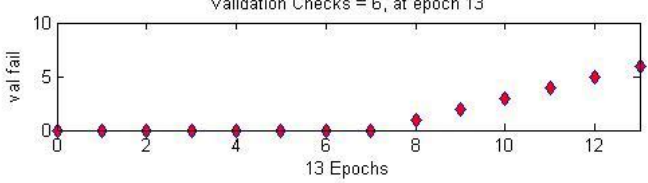

\section{CONCLUSION}

On the basis of this model it very accurate to determine the loss of packets. This system uses all most important features of a packet and all factors having some participation of them.

Besides this much work can be done one can use more number of input values it is a hybrid judgment intuitive and inference. Which may bias some time but not. One can use different training algorithm also use some other methods rather than centroid.

\section{REFERENCES}

[1] B. Braden, D. Clark, J. Crowcroft, B. Davie, S. Deering, D. Estrin, S. Floyd, V. Jacobson, G. Minshall, C. Partridge,L. Peterson, K. Ramakrishnan,S. Shenker, J.Wroclawski, \& L. Zhang, Recomedations on Queue Management and Congestion Avoidance in the Internet,RFC2309, April 1998.

[2] C. Hollot, V. Misra, D. Towsley, and W. Gong. A Control Theoretic Analysis of RED. in Proceedings of IEEE INFOCOM'01.Anchorage, Alaska, USA, April 2001,1510-1519.

[3] C. Hollot, V. Misra, D. Towsley, and W. Gong. On designing Improved Controllers for AQM Routers Supporting TCP Flows. in Proceedings of IEEE INFOCOM'01, Anchorage, Alaska, USA, April 2001,1726-1734.

[4] David Lapsley and Steven Low, Random Early Marking for Internet Congestion Control[A], in Proceedings of IEEE Globecom'99, Rio de Janeiro.Brazil,December $1999,1747-1752$.

[5] F.Anjum and L.Tassiulas, Balanced-RED:An Algorithm to Achieve Fairness in Internet. http://www.isr.umd.edu/CSHCN/

[6] Lin, D. and Morris, R., Dynamics of random early detection,in Proceedings of ACM SIGCOMM'97,Cannes, France, October 1997,127-137.
[7] M. May, J. Bolot, C. Diot, and B. Lyles. Reasons Not to Deploy RED. in Proceedings of 7th. International Workshopon Quality of Service (IWQoS'99), London, UK, June 1999, 260-262.

[8] Munron.Modern Approaches to Control System Design.New York,Prentice-Hall,Inc.1979.

[9] Ott, T., Lakshman, T. and Wong, L., SRED: Stabilized RED, in Proceedings of INFOCOM'99, New York, NY, March 1999, 1346-1355.I

[10] Pan, R., Prabhakar, B. and Psounis, K., CHOKe - A Stateless Active Queue Management Scheme For Approximating Fair Bandwidth Allocation,in Proceedings of INFOCOM'00, Tel-Aviv, Israel, April 2000, 942-951.

[11] Ren Fengyuan, Lin Chuang, Ying Xunhe, Shan Xiuming, Wang Fubao.A Robust Active Queue Management Algorithm Based on Sliding Mode Variable Structure Control. in Proceedings of INFOCOM'02,New York,USA,June 2002,64-79.

[12] R. Mahajan, S. Floyd, D. Wetheral. Controlling HighBandwidth Flows at Congested Router, in Proceedings of

[13] S. Floyd, and V. Jacobson, Random Early Detection Gateways for Congestion Avoidance, IEEE/ACM Transactions on Networking, vol. 1 no. 4, August 1993, 397-413.

[14] S. Floyd, Recommendations on using the gentle "variant of RED”,http://www.aciri.org/floyd/red/gentle.html.

[15] S. Floyd, R. Gummadi, and S. Shenker. Adaptive RED: an algorithm for increasing the robustness of RED's Active Queue Management.http://www.icir.org/ floyd.

[16] Vishal Misra, Wei-Bo Gong, and Don Towsley,Fluidbased Analysis of a Network of AQM Routers Supporting TCP Flows with an Application to RED,in Proceedings of ACM SIGCOMM'00, Stockholm, Sweden,August 2000,151-160

[17] Bonissone, P.P. and Badami, V. and Chiang, K.H. and Khedkar, P.S. and Marcelle, K.W. and Schutten, M.J., "Industrial applications of fuzzy logic at General Electric", Proceedings of the IEEE, 1995, Volume 83, 450--465

[18] Boskovitz, V. and Guterman, H., "An adaptive neurofuzzy system for automatic image segmentation and edge detection", Fuzzy Systems, IEEE Transactions on, 2002, Volume 10, 247-262

[19] Klement, E.P. and Slany, W., "Fuzzy logic in artificial intelligence", Christian Doppler Laboratory Technical Reports, 1994, Volume 67 